\title{
W-Band Sheet Beam Klystron Simulation
}

\author{
E. R. Colby ${ }^{\ddagger}$, G. Caryotakis ${ }^{\ddagger}$, W. R. Fowkes ${ }^{\ddagger}$, D. N. Smithe ${ }^{\dagger}$ \\ ${ }^{*}$ Stanford Linear Accelerator Center ${ }^{1} 2575$ Sand Hill Rd. Menlo Park, CA 94025 and \\ ${ }^{\dagger}$ Mission Research Corporation 8560 Cinderbed Road Ste. 700, Newington, VA 22122
}

\begin{abstract}
With the development of ever higher energy particle accelerators comes the need for compactness and high gradient, which in turn require very high frequency high power if sources. Recent development work in W-band accelerating techniques has spurred the development of a high-power W-band source. Axisymmetric sources suffer from fundamental power output limitations $\left(\mathrm{P}_{\mathrm{sat}} \lambda^{2}\right)$ brought on by the conflicting requirements of small beam sizes and high beam current. The sheet beam klystron allows for an increase in beam current without substantial increase in the beam current density, allowing for reduced cathode current densities and focussing field strengths. Initial simulations of a 20:1 aspect ratio sheet beam/cavity interaction using the 3 dimensional particle-in-cell code Magic3D have demonstrated a $35 \%$ beam-power to RF power extraction efficiency. Calculational work and numerical simulations leading to a prototype $\mathrm{W}$-band sheet beam klystron will be presented, together with preliminary cold test structure studies of a proposed RF cavity geometry.
\end{abstract}

\section{INTRODUCTION}

The construction of a $5 \mathrm{TeV}$ electron collider will remain impractical unless the energy gain per unit distance can be increased by an order of magnitude or more over the present value. Simple arguments concerning RF structure breakdown and considerations of electron trapping ("dark current" formation) lead to higher RF frequencies as the choice for high gradient acceleration (1) in conventional structures.

Consequently, a research program to explore the issues involved in fabricating and using accelerator structures at $91.392 \mathrm{GHz}$ (32 times the SLAC frequency of 2856 $\mathrm{MHz}$ ) was begun in 1996(2). Given fill times and shunt impedances that can be expected of conventional w-band accelerating structures, 10-20 nanosecond RF pulses of several hundred megawatts peak power will be needed. Actively switched structures(3) will require 50-100 nanosecond RF pulses of $\sim 10$ megawatts, owing to the high pulse compression factors expected.

RF cavity dimensions within a klystron scale with the wavelength of the produced radiation as must the beam dimensions. Given an upper limit on cathode current density (presently 10-20 A/cm ${ }^{2}$ ), this implies the beam current, and therefore the output power, must scale like $\lambda^{2}$. If one further requires that the electron gun operate at the space

\footnotetext{
${ }^{1}$ This effort is funded by the AFOSR MURI on High Energy Microwave Sources and by DOE contract number DE-AC02-76SF00515.
} 
charge limit and at the same current density loading, the gun voltage must scale like $\lambda^{4 / 3}$, making the overall output power scaling $\lambda^{10 / 3}$.

Scaling the state-of-the-art SLAC 75 MW X-band klystron to W-band under these conditions implies a peak output power of only $73 \mathrm{~kW}$. Following the scaling, the beam current would be only 4 Amperes, with the perveance, which does not scale with wavelength at all, remaining unchanged. As the bunching efficiency scales like $1 /(1+\mu \mathrm{P} / 1.33)$ (4) one expects that the tube efficiency at $\mathrm{W}$-band, under the scaling, would be the same as the X-band tube, allowing little room for improvement.

Several alternatives exist, each increasing the beam current without increasing the perveance, including the annular beam klystron (ABK), multiple beam klystron (MBK), and sheet beam klystron (SBK). Of the alternatives, the MBK and SBK are being pursued at the present time in a joint SLAC/MRC effort, with the MBK being described elsewhere(5). The basic idea driving all three schemes is the increase of the beam current without an increase of the number density, implying more beam power without an increase in the space charge forces which degrade tube efficiency. The ABK and SBK both increase the beam cross section area while using a single focussing channel and RF circuit, while the MBK adds more beamlets in separate focussing channels, but still in a common RF circuit. The complexities of having many focussing channels in the MBK is, in some sense, balanced by the complexities of focussing annular or sheet beams.

\section{Background}

The sheet beam klystron (or "waveguide klystron") is an old idea dating back to the 1950s that received renewed attention in the 1980s as a candidate X-band power source for the Next Linear Collider (NLC). Is was determined at the time that beams of interesting power levels (200 MW) could not be transported without significant $(50 \%$ or more) beam losses $(6,7,8)$, and the SBK fell into disfavor for the X-band application. For the present application, we will be considering much lower beam currents.

Outstanding issues for the sheet beam klystron arise from the unusual geometry of the RF cavities and the beam. In particular:

1) Beam edges in the large dimension experience strongly nonlinear space charge fields and tend to erode rapidly;

2) Cavity shunt impedance becomes rapidly worse with increasing transverse dimension aspect ratio, and TM110 mode spacing decreases with increased gap width, causing mode competition problems;

3) The drift tubes between cavities are no longer cutoff at the klystron frequency, inviting instability and oscillation. 
Each of these issues will be dealt with in the next section. In addition, several issues arise from the small working wavelength of the klystron:

4) Dimensional tolerances are generally $\sim 10^{-3} \lambda$, which at millimeter wavelengths, requires micrometer tolerances;

5) Conventional brazing techniques produce fillets of braze material within the RF cavities that are large enough to cause serious detuning at millimeter wavelengths, requiring use of either minimal brazing material or diffusion bonding techniques.

Industry has pursued the manufacture of mm-wave and sub-mm-wave components for some years, with the production of $50 \mathrm{GHz}$ microstrip components for telecommunications, and $77 \mathrm{GHz}$ components for automotive radar sensors(9), micromachined W-band RF filters (10), and tapered-rod antennae at frequencies up to $850 \mathrm{GHz}(11)$, providing just a few examples.

Fabrication of 1-,7-, 25- and 40-cell muffin tin structures at $91.392 \mathrm{GHz}$ using wire EDM and diffusion bonding techniques has been underway at SLAC for nearly two years, with encouraging results(12,13). In addition, LIGA, a deep x-ray lithography technique, has been used to produce 66-cell constant gradient structures at 94 and 108 $\mathrm{GHz}$ with results that are becoming excellent mechanically (14) and encouraging electrically $(14,15)$.

\section{Sheet Beam Focussing}

Sheet beam transport in a solenoidal field is known to be unstable(16), requiring other focussing magnet configurations to be considered. Quadrupole focussing is one option, however for the small, high-aspect ratio beams, and short period lengths required, achieving the required field strengths will be difficult.

Permanent magnet focussing offers both energy efficiency, and substantial field strength from a small source. Periodic Cusp Magnetic (PCM, more commonly "PPM") focussing and Magnetic Deflection ("Sturrock" or "Wiggler") focussing have both been considered as they are (1) amenable to high field strengths when realized with permanent magnets, (2) immune to low frequency AC instabilities.

Erosion of the edges of the beam in the large dimension takes place on a time scale set by the local horizontal electric field strength, generally within a factor of two of the plasma period, hence significant edge erosion is not expected over the length of the prototype klystron, only approximately one plasma wavelength in overall length. 
Beam transport will be stable in a PPM lattice so long as focussing strength exceeds the space charge forces pushing the beam apart, and as long as the phase advance per unit focussing cell is less than $90^{\circ}$ :

$$
2 \omega_{p}^{2} \leq \Omega_{0}^{2} \leq 2 k_{m}^{2} u_{0}^{2}
$$

with $k_{m}=2 \pi / L_{m}$ the PPM lattice wavenumber, and $u_{0}$ the beam velocity. For the proposed beam parameters (given in table 2 below), this means:

$$
2.04 \leq B_{0}^{2}\left[k G^{2}\right] \leq 50.3 \rightarrow 1.43 k G \leq B_{0} \leq 7.1 k G
$$

Figure 1 below gives an example of a PPM focussing unit cell, assuming $\mathrm{NbFeB}$ permanent magnet material in 6x3 mm (length $\mathrm{x}$ depth) blocks and 1008 low carbon steel pole pieces in $4 \times 3 \mathrm{~mm}$ blocks, offset $1 \mathrm{~m}$ toward the beam axis centerline. The on axis field strength, by PANDIRA calculation, is $4.9 \mathrm{kG}$, or more than a factor of three above the lower stability limit. The substantial leakage flux on the outside of the PPM magnet stack will permit permeable shunt tuning of the lattice once assembled.

Although figure 1 (left) below clearly shows that the focussing field $\mathrm{Bz}(\mathrm{z})$ has substantial spatial harmonic content beyond the fundamental, the klystron simulations kept only the fundamental space harmonic, underestimating the true focal strength by some factor less than two. Booske's form for the fields is employed, together with a small horizontally focussing quadrupole term (which can be derived by pole tip shaping):

$$
\begin{aligned}
& B x(x, y, z)=-B_{0}\left(k_{x} / k_{m}\right) \sinh \left(k_{x} x\right) \cosh \left(k_{y} y\right) \cos \left(k_{m} z\right)-k_{q} y \\
& B y(x, y, z)=-B_{0}\left(k_{y} / k_{m}\right) \cosh \left(k_{x} x\right) \sinh \left(k_{y} y\right) \cos \left(k_{m} z\right)-k_{q} x \\
& B z(x, y, z)=B_{0} \cosh \left(k_{x} x\right) \cosh \left(k_{y} y\right) \sin \left(k_{m} z\right)
\end{aligned}
$$

Wiggler focussing is an alternative to PPM and has fields of the form (17):

$$
\begin{aligned}
& B x(x, y, z)=0 \\
& B y(x, y, z)=B_{0} f(z) \cosh \left(k_{w} y\right) \sin \left(k_{w} z\right) \\
& B z(x, y, z)=B_{0} f(z) \sinh \left(k_{w} y\right) \cos \left(k_{w} z\right)
\end{aligned}
$$

where $k_{w}$ is the wiggler wavenumber, and the tapering function $f(z)$ at the wiggler entrance is:

$$
f(z)=\sin ^{2}\left(k_{w} z / 4 N_{t}\right)
$$


with $N_{t}$ counting the length of the taper in wiggler periods. As with the PPM focussing, astute pole tip shaping can be used effectively to provide force balance in both horizontal and vertical planes.
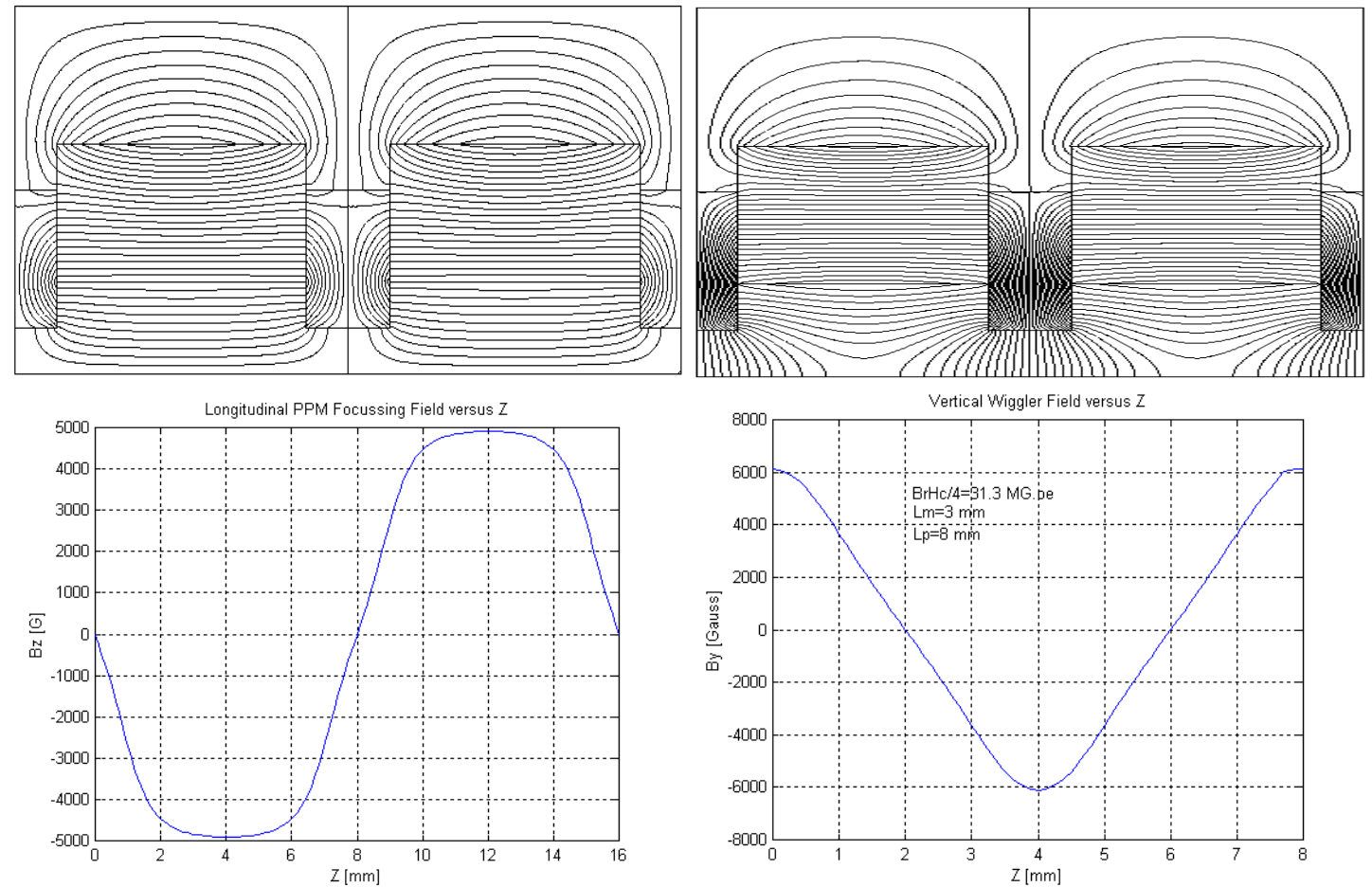

FIGURE 1. PPM cell fields for $16 \mathrm{~mm}$ period length (left) and wiggler cell fields for $8 \mathrm{~mm}$ period length (right). Plotted fields are well above the stability threshold fields in each case, indicating that meeting the stability requirement is straightforward.

For Wiggler focussing to be stable, the same force balance condition must be met as with the PPM lattice, but owing to the different spatial wavenumber controlling the vertical focussing (it is $k_{w}=2 \pi / L_{w}$, which scales like the wiggle period length, rather than $k_{y}$, which scales like the vertical PPM magnet dimension), the condition is (18):

$$
\omega_{p}<\sqrt{\frac{\gamma}{2}} \Omega_{w} \rightarrow 1.0 k G<B_{w}
$$

for the present case, with $\Omega_{w}=e B_{w} / m_{e}$. As with the PPM focussing, the basic focussing mechanism is pondermotive: the beam is pushed towards the point of minimum magnetic field energy density by a force proportional to the gradient in $|\mathrm{B}|^{2}$. For the PPM focussing the variation was fast, scaling with the vertical height of the PCM magnet gap. For the wiggler focussing, the fields vary much more slowly in the vertical plane, allowing a much larger matched vertical beam size. Figure 1 above (right) shows the magnetic field strength for a wiggler focussing cell with an $8 \mathrm{~mm}$ cell length. 
Additional beam area convergence is possible by appropriate tapering of the entrance fields of the transport lattice. By adding a matching telescope (a quadrupole doublet) near the start of the focussing lattice, it is possible to further reduce the vertical beam size by a useful amount, easing the cathode current density loading. Figure 2 below shows both analytic (left) and 3D PIC simulation (right) of 4:1 vertical compression and subsequent match to a wiggler lattice.
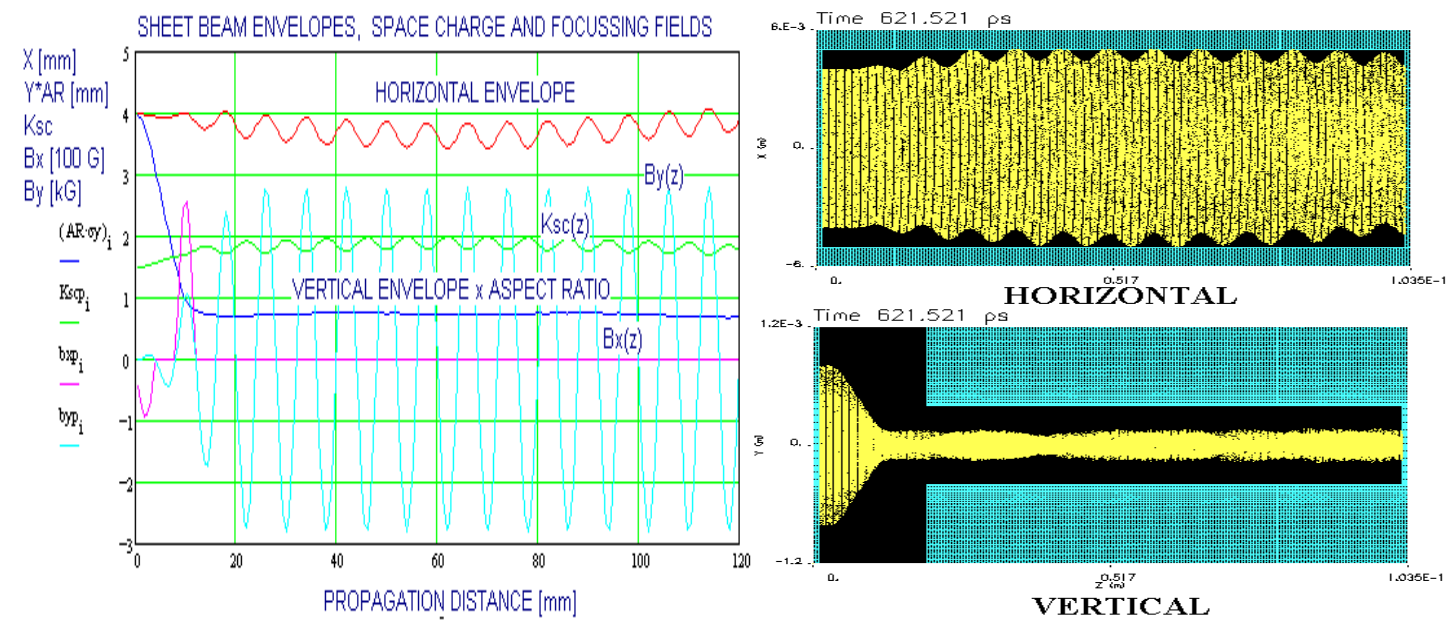

FIGURE 2. Envelope integration (left) and 3D PIC simulation (right) of compressed, taper matched, wiggler focussed sheet beam showing 4:1 vertical compression

\section{Klystron Waveguide Cavity Design}

The conventional modes used for klystron cavities, the $\mathrm{TM}_{010}$ mode of the cylindrical pillbox, and its Cartesian analog, the $\mathrm{TM}_{110}$ mode of the rectangular pillbox, both have electric fields which vary rather rapidly off the cavity axis. This would give rise to uneven bunching of a sheet beam, and make impossible a uniform impedance match between beam and output cavity for maximum efficiency. Alternative cavity geometries must therefore be considered.

Several high aspect ratio cavity geometries have been proposed for the SBK. (19) The candidate considered here is a variant of the "barbell" cavity proposed by Miller(20). It is simply a section of slotted waveguide operating in the $\mathrm{TE}_{10}$ mode just above cutoff, hence $\beta z \approx 0$, Ez does not vary along the guide length, and the guide impedance is very high. The cutoff waveguide is terminated in a $\lambda / 4$ waveguide section at each end to match between the high impedance cutoff waveguide and the shorting plane at the coupling aperture. In practice the matching cavities at each end are somewhat shorter than $\lambda / 4$ due to the coupling aperture in the outer wall.

A cold test model of a non-reentrant slotted waveguide cavity was built at X-band to test the field flatness and electrical properties of the proposed geometry. The R/Q 
profile was deduced by resonant perturbation methods, using a dielectric rod inserted though the cavity beam tube and is shown in figure 3 below.
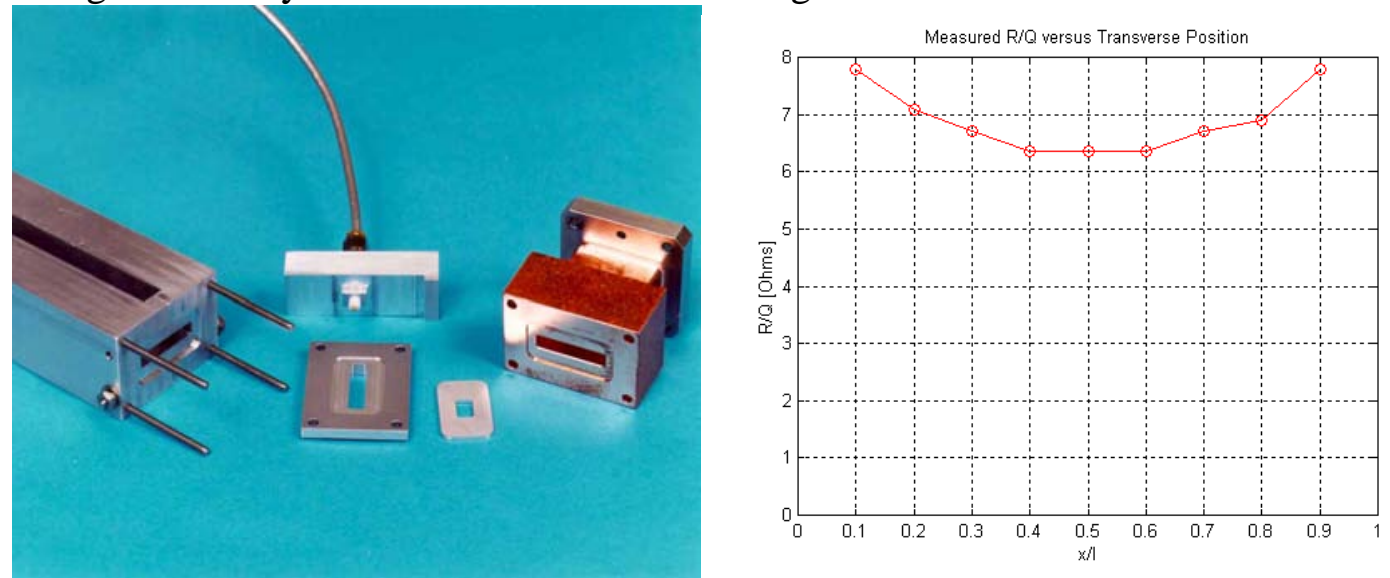

FIGURE 3. Non-reentrant 20:1 X-band waveguide cavity cold test model, showing cutoff waveguide section (left) [beam opening faces upward], $\lambda / 4$ cavity and coupling iris (center), capacitive field probe (top center) and waveguide (right), and measured R/Q of model.

With a low R/Q, design of an efficient output circuit becomes more difficult, requiring travelling wave output structure to raise the shunt impedance high enough to give good coupling to the beam. Efforts to improve the R/Q of the structure were clearly indicated.

A simple extension of the barbell cavity involves substituting a slotted, ridged waveguide for the slotted rectangular guide of the barbell cavity. By making the cavity re-entrant in this fashion, it becomes possible to dimension the cavity and gap independently, allowing gap coupling constant considerations to set the gap length, and cavity $\mathrm{Q}$ and R/Q considerations to dimension the cavity proper. The ridged waveguide, like the rectangular waveguide, must be operated close to cutoff to give good field flatness, but this is not difficult, owing to the large slot cut for the beam tube, which tends to compensate the increase in capacitance from the reentrancy. The addition of reentrant "noses" to the structure is a complication which changes only the cutoff waveguide cross section profile, and thus is not significantly more difficult to fabricate.

The computed field profile $\mathrm{Ez}(\mathrm{x}, \mathrm{y})$ for a 20:1 aspect ratio re-entrant waveguide cavity is shown in figure 4 below.

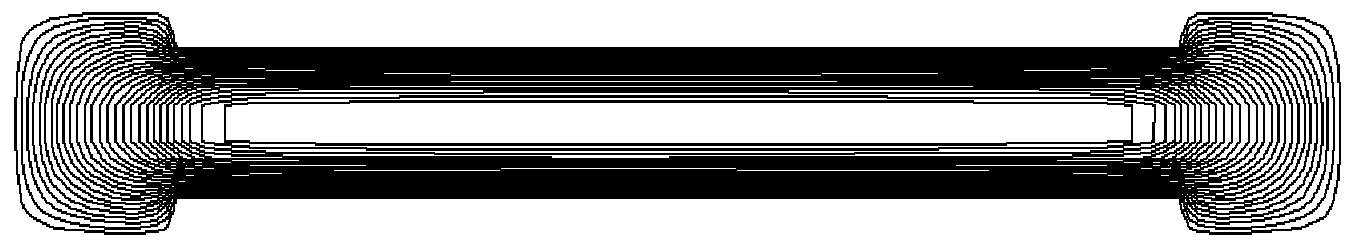

FIGURE 4. Contour plot of $\mathrm{Ez}(\mathrm{x}, \mathrm{y})$; the beam motion is into the page. 
Parameters of this 20:1 cavity are summarized in table 1 below. The R/Q of the waveguide cavity varies inversely with the aspect ratio of the "flat-field" region,

TABLE 1. Reentrant 20:1 aspect ratio cavity properties.

\begin{tabular}{l|l|l}
\hline Parameter & Value & \\
\hline Frequency, $\mathrm{TM}_{110}$ & $91.392 \mathrm{GHz}$ & \\
Nearest mode $\mathrm{TM}_{310}$ & $95.5579 \mathrm{GHz}$ & $\mathrm{TM}_{210}$ cannot couple to source \\
Mode Separation $\left(\Delta \mathrm{f}^{*} \mathrm{Q}_{\mathrm{o}} / \mathrm{f}_{\mathrm{o}}\right)$ & $>50$ & $\mathrm{TM}_{310}-\mathrm{TM}_{110}$ \\
$\mathrm{Q}_{\mathrm{o}}$ & 1172 & See text \\
$\mathrm{R} / \mathrm{Q}$ & $19.4 \Omega$ & For $\beta=\infty, \mathrm{x}=\mathrm{y}=0$ particle \\
$\mathrm{R} / \mathrm{Q} / \square$ & $388 \Omega / \square$ & \\
Aspect Ratio & $20: 1$ & \\
\hline
\end{tabular}

implying that a 40:1 cavity would have approximately half the R/Q value of the 20:1 cavity shown. Consequently, the invariant quantity " $\mathrm{R} / \mathrm{Q} / \square$ ", defined as the product of the aspect ratio and the $\mathrm{R} / \mathrm{Q}$, is also given below as a more generally applicable figure of merit.

The main virtue of the SBK is that the beam current may be increased beyond the round beam value without increasing the beam perveance and lowering the tube efficiency. Thus, the wider the beam, the more output power may be obtained. Several practical constraints must be considered which impose a limit to the beam width.

One limit on the length of the cutoff waveguide section of the barbell cavity is imposed by mode competition from the adjacent cavity modes, which grow closer as the cutoff section grows longer:

$$
\Delta k \equiv k_{m}-k_{n}=\frac{m \pi}{L x}-\frac{n \pi}{L x} \propto \frac{1}{L x}
$$

Clearly as the horizontal length Lx becomes greater, the separation between $\mathrm{TM}_{\mathrm{n} 10}$ modes decreases. A practical length limit is therefore determined by the requirement that the $\mathrm{TM}_{110}$ and $\mathrm{TM}_{210}$ modes be separated by at least the twice the sum of their respective $3 \mathrm{~dB}$ loaded bandwidths (200-500), requiring:

$$
L x \leq \frac{Q c}{2 f_{o}} \approx 33 \mathrm{~cm}
$$

A far stricter limitation comes from the requirement of field flatness in the cutoff waveguide. Impedance tapering the cutoff waveguide section(21) is one possible way to relax this constraint. 


\section{Oscillation/Mode Leakage Issues}

The drift spaces between the cavities for the SBK have dimensions that permit the TEn0 modes to propagate at the klystron operating frequency. For the chosen 20:1 aspect ratio, the first six modes, $\mathrm{TE}_{10}$ through $\mathrm{TE}_{60}$, all are above cutoff at the klystron operating frequency. Although none of these modes is capable of being driven by a symmetric $\mathrm{TM}_{110}$ cavity mode, imperfections of the real klystron cavities will cause some coupling to each of these modes. Common to all the TE modes are substantial wall currents flowing in the narrow wall of the waveguide. Consequently, slotting the drift tube on the narrow walls, and mounting of an RF absorber such as SiC or lossy ceramic in the slots will suppress these modes, and is the solution adopted here. Yu and Wilson(20) proposed and numerically demonstrated that choke cavities could effectively suppress the communicating modes of the drift tubes on a mode-by-mode basis, but this scheme requires the addition of another set of cavities to the klystron that must be tuned, and is quite mode-specific, whereas slotting the drift tube walls damps all modes with appreciable wall currents in the vicinity.

Additional damping can be provided by lining the top and bottom surfaces of the drift tube with lossy conducting material, which will further increase the attenuation of the drift tube modes.

\section{KLYSTRON SIMULATION}

Small-signal analytic treatment using a MathCAD program developed by SLAC for round beam klystrons and modified for sheet beam klystrons was used to establish rough values for optimal cavity $\mathrm{Q}$, frequency and placement for the chosen beam aspect ratio, voltage and current.

Optimal cavity performance was tuned up using the 3D finite difference EM solver GdfidL, solved again (in Magic3D) in the time domain by Green function techniques to establish the "hot" cavity properties, and then coded into a Magic3D simulation of the klystron, beginning from the focal waist after the electron gun. Detailed parameters for the design prototype are shown in table 2 below.

A proof-of-principle numerical experiment to test the possibility of extracting significant RF power from a sheet beam in a waveguide output cavity was carried out. A $15 \mathrm{~A}, 140 \mathrm{kV}$ sheet beam was imprinted with an ideal amplitude RF current modulation, then allowed to interact with a waveguide cavity. The cavity was loaded with lossy material at the position of the coupling apertures, to achieve the same general field shape and Q as could be expected from a waveguide-coupled cavity. The kinetic energy as a function of $\mathrm{z}$ and the power extracted (i.e. the power loss in the a 
Table 2. Target parameters for sheet beam klystron design.

\begin{tabular}{l|l|l}
\hline Parameter & Value & Comments \\
\hline Center frequency & $91.392 \mathrm{GHz}$ & $32 \mathrm{xSLAC}$ \\
Bandwidth & $70 \mathrm{MHz}$ & $1 \mathrm{~dB} \mathrm{BW}$ \\
\# Cavities & 3 & \\
Small-signal Gain & $25 \mathrm{~dB}$ & $150 \mathrm{kV}$ max \\
Beam Voltage & $140 \mathrm{kV}$ & \\
Beam Current & $15 \mathrm{~A}$ & \\
Perveance & $0.29 \mu \mathrm{P}$ & $20: 1$ aspect ratio \\
Beam Dimensions & $0.4 \times 8 \mathrm{~mm}$ & \\
Perveance per Square & $14.4 \mathrm{nP} / \square$ & \\
Beam Power & $2.25 \mathrm{MW}$ & \\
Current Density & $469 \mathrm{~A} / \mathrm{cm}^{2}$ & \\
Cathode Current Density & $4.69 \mathrm{~A} / \mathrm{cm}^{2}$ & \\
Gun Compression Ratio & $25: 1$ & Wiggler $+0.05 \mathrm{~T} / \mathrm{m}$ HF quadrupole \\
Matching Comp. Ratio & $4: 1$ & \\
Confining field Peak & $1550 \mathrm{G}$ & \\
PCM Lattice wavelength & $8 \mathrm{~mm}$ & Re-entrant geometry \\
Plasma Wavelength & $75 \mathrm{~mm}$ & \\
Cavity R/Q & $19.4 \Omega$ & \\
Drift Tube Dimensions & $0.6 \mathrm{x} 10.0 \mathrm{~mm}$ & \\
\hline
\end{tabular}

aperture load) are shown in figure 5 below. A power extraction efficiency of $35 \%$ was observed in the best case, providing evidence that efficient power extraction from a sheet beam is possible with the proposed waveguide cavity geometry.
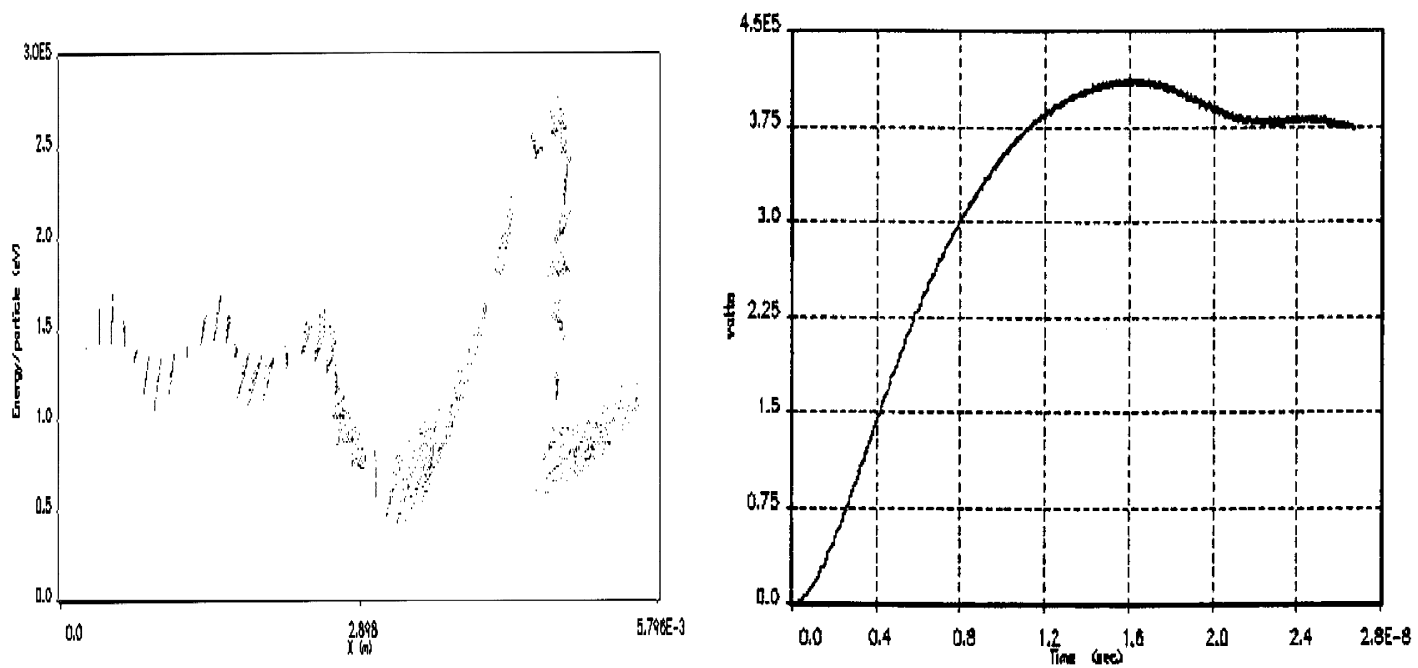

FIGURE 5. Ideally bunched beam interacting with a loaded waveguide cavity. Kinetic energy as a function of propagation distance (left) and ohmic power loss in one of two aperture loads as a function of time. The true output power would be twice the value shown. 


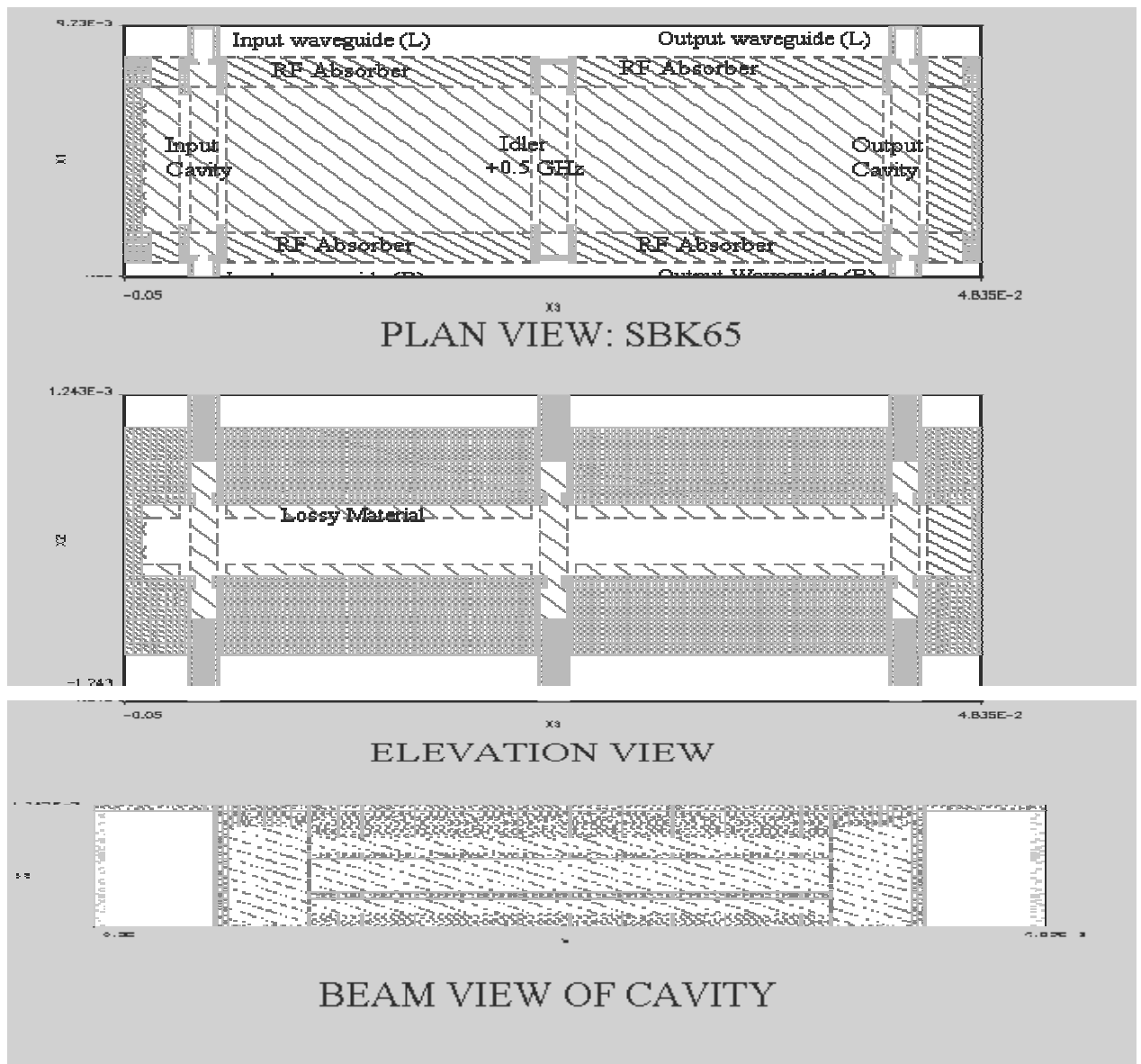

FIGURE 6. Geometry of 3-cavity sheet beam klystron for the Magic3D simulations.

As can be seen in figure 6, several measures have been taken to curb oscillations, both real, and numerical in origin. The drift tubes have been slotted and an ideal absorber inserted in the gap to dampen the TE modes. The top and bottom surfaces of the drift tubes have been loaded with lossy material to dampen higher-order TM modes which would be otherwise excited by numerical noise. The drift space beyond the output cavity has likewise been loaded with an ideal RF absorber to catch the initial plane wave launched when the electron beam is turned on, and to provide further dampening for the drift tube modes.

Cavity Qs are set by the conductance of the lossy material within each cavity, and by the size of the coupling aperture (input and output cavities only). Coupling aperture dimensions are set by observing the gap voltage, wall loss power, and drive power (through the waveguide) to the cavity, and inferring the respective Qs from a knowledge of the cavity R/Q.

Drive power to the input is established by enforcing a normalization on a line spanning the input waveguide parallel to the guide electric field. 
Electrons are emitted starting at what would be the beam waist following the electron gun for the klystron and transported in a wiggler lattice to a conducting wall downstream of the output cavity. Properties of the focussing optics are given in table 3 below.

TABLE 3. Wiggler focussing parameters.

\begin{tabular}{ll}
\hline Parameter & Value \\
\hline Wiggler Period & $8 \mathrm{~mm}$ \\
Number of periods & 6 \\
Taper length & 2 periods \\
On-Axis Induction & $1150 \mathrm{Gauss}$ \\
Pole Gap (vertical) & $1.5 \mathrm{~mm}$ \\
Background Quad & horizontally focussing \\
Back. Quad gradient & $10 \mathrm{~T} / \mathrm{m}$ \\
\hline
\end{tabular}

The difficulties involved in constructing a full 3D simulation of the RF circuit for the SBK are such that at this time only a poorly optimized klystron example can be given. Figure 7 below shows the small-signal (see, for example (21)) performances for both the optimized 3-cavity klystron (which assumes an extended output circuit) and the present case, showing that there is substantial room for improvement. The largest improvement will come from incorporating the extended output circuit, correcting the errant cavity Qs and coupling $\beta$ s, and adjusting the wiggler focussing to give more even beam transport.
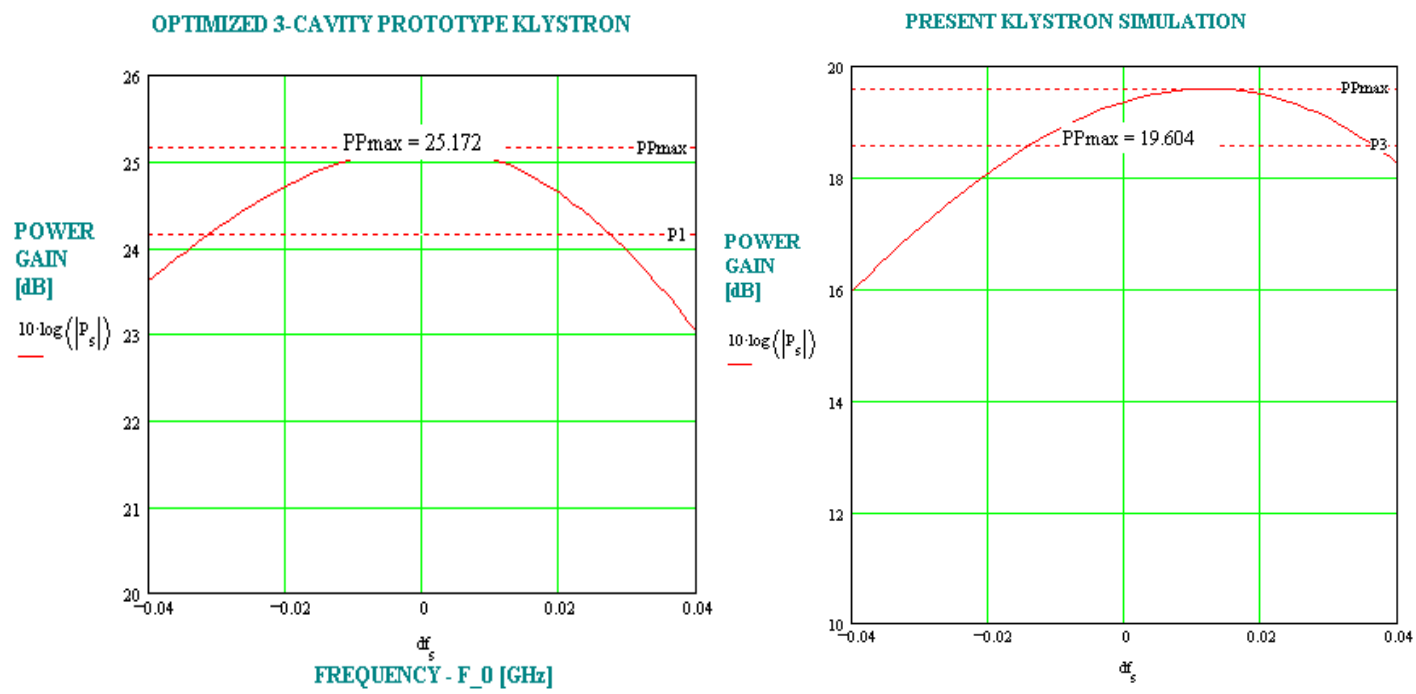

FIGURE 7. Optimal, and current simulation expected performances for the 3 -cavity sheet beam klystron.

Figure 8 below shows the beam bunching in the horizontal and vertical planes, with the vertical focussing effects of the barbell cavity plainly visible. The overfocussing in the vertical plane occurs for much lower (factor of 4) wiggler field strength than one expects by straightforward integration of the beam envelope equations (22), providing 
evidence that the vertical space charge fields are inadequately represented in the simulation.

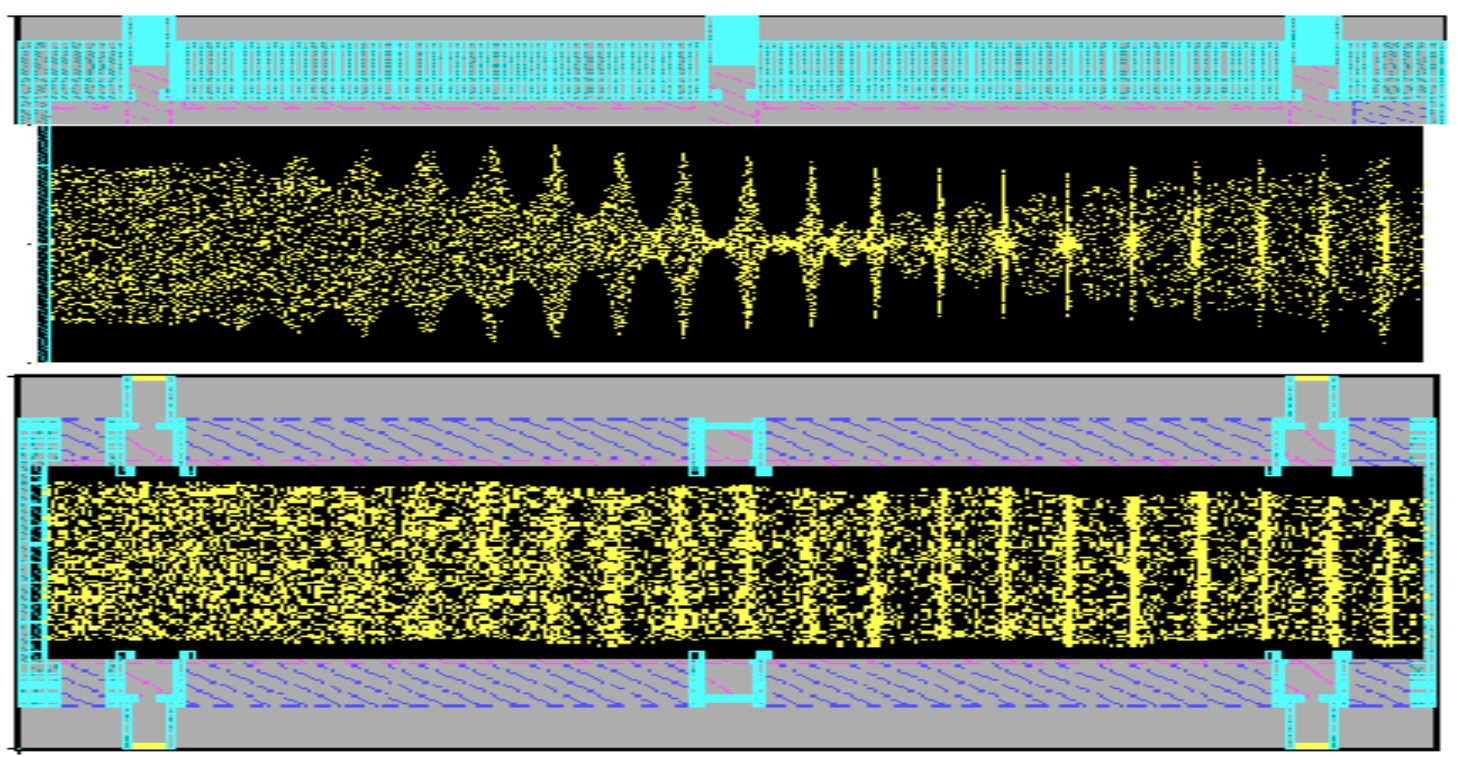

FIGURE 8. Beam bunching for the 3-cavity SBK in the horizontal (top) and vertical (bottom) planes.

Power input and output, as measured at a plane (different from the port plane) in the entrance and exit waveguides, respectively, is shown in figure 9 below. Two waveguides connect to each of the input and output cavities, so the actual power values are twice those shown.
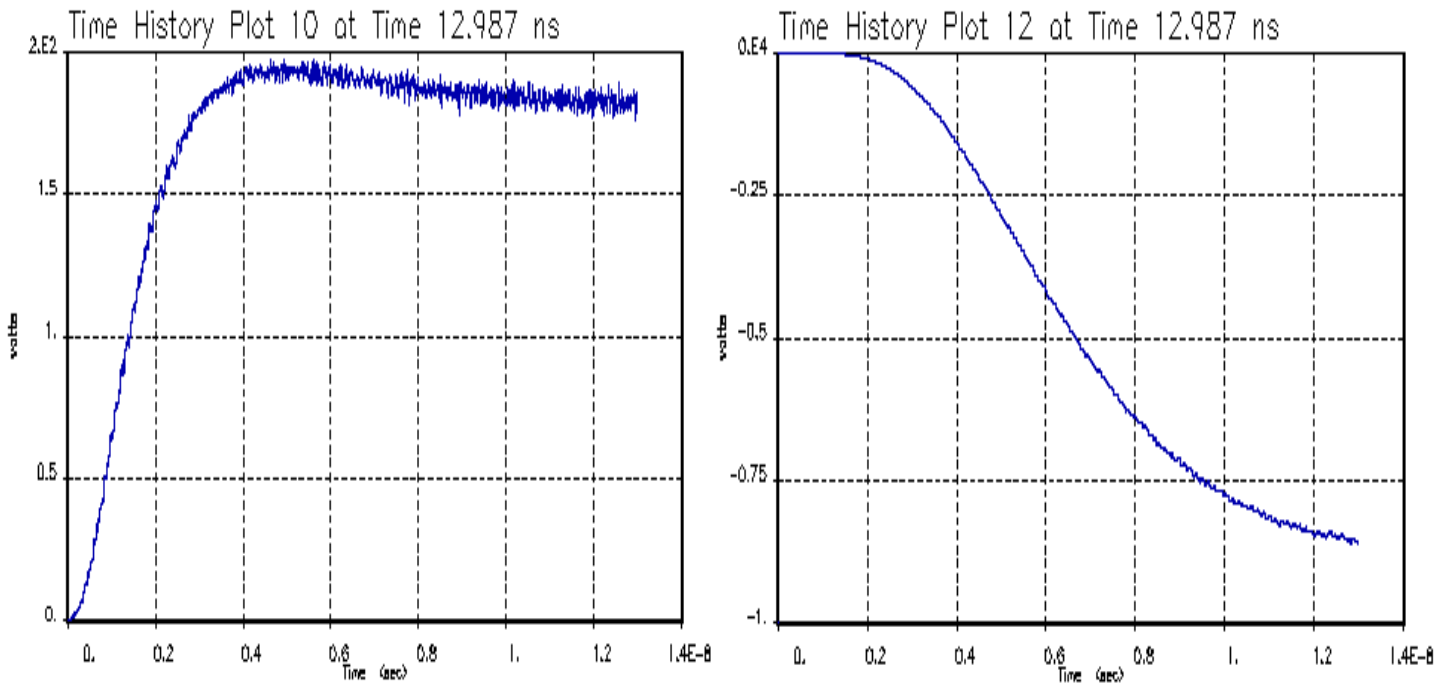

FIGURE 9. Input and output power to 3-cavity SBK. Actual input and output powers are twice those shown. Data shown required 4 days of computation time to produce. 
The output power of $17.5 \mathrm{~kW}$ versus $370 \mathrm{~W}$ input gives a power gain of only $16.7 \mathrm{~dB}$, and a negligible power efficiency. But as shown in the cavity tuning table below, there is clearly room for improvement. Cavity parameter values for the simulation are deduced from power dissipations, gap voltages and the cavity's computed R/Q.

TABLE 4. Cavity parameters for 3-cavity SBK, both optimized case (in a small signal sense) and the Magic3D simulated case.

\begin{tabular}{l|l|l|l|l|l}
\hline $\begin{array}{l}\text { Parameter } \\
\text { Optimized }\end{array}$ & $\begin{array}{l}f_{\mathrm{o}} \\
{[\mathrm{GHz}]}\end{array}$ & $\mathrm{Qo}$ & $\mathrm{Qe}$ & $\begin{array}{l}\mathrm{R} / \mathrm{Q} \\
{[\Omega]}\end{array}$ & $\begin{array}{l}\mathrm{drift} \\
{[\mathrm{mm}]}\end{array}$ \\
\hline Input & 91.392 & 1200 & 400 & 20 & 24 \\
Idler & 91.405 & 1200 & $\infty$ & 20 & 24 \\
Output & 91.392 & 1200 & 835 & 90 & 18 \\
Simulated & 91.41 & 2590 & 1050 & 20 & 18 \\
Input & 91.46 & 16288 & $\infty$ & 20 & \\
Idler & 91.41 & 2502 & 1090 & 20 & \\
Output & & & & & \\
\hline
\end{tabular}

The bunching shown in figure 10 below is computed by integrating the current density across a plane perpendicular to the axis of the klystron, in a manner reflective of how the beam would interact with a waveguide cavity at that point. Any curving of the space charge waves due to the wiggler focussing, space charge effects or nonuniformity of the RF fields in the upstream cavities would reduce the observed RF current component.

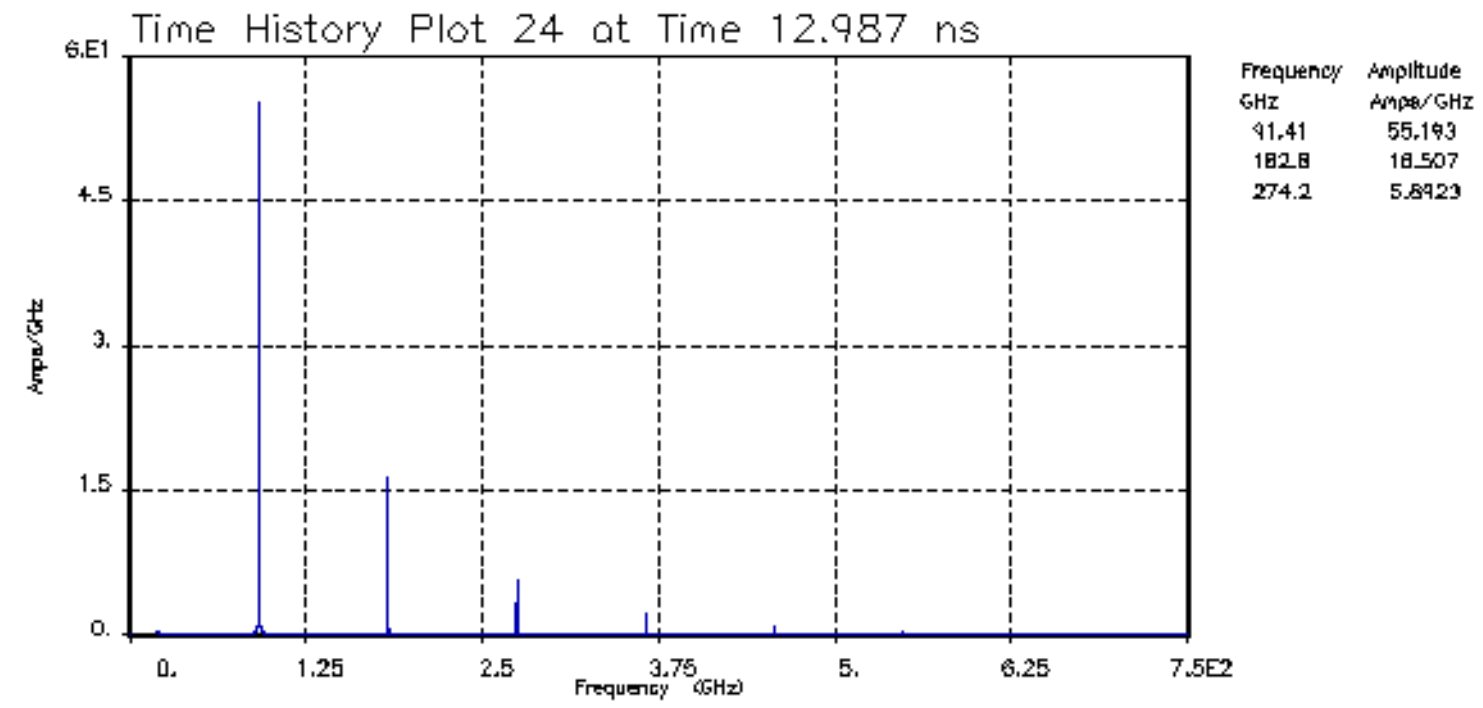

FIGURE 10. Bunching harmonic amplitudes $2 \mathrm{~mm}$ upstream of the output cavity.

The dominant reason for the poor efficiency of the simulation klystron is the substantial mismatch between the output gap impedance and the beam impedance. For the given parameters, the output gap impedance should ideally be(25): 


$$
Z_{g a p}=Q_{e}(R / Q)=\frac{V_{o}}{I_{o}} \frac{1}{M^{2} \frac{I_{v f}}{I_{d c}}}=61 \mathrm{k} \Omega
$$

whereas the simulation klystron gap impedance is $Q_{e}(R / Q)=21 \mathrm{k} \Omega$, too low by a factor of three. This low impedance causes the gap voltage to be too low to effectively extract the beam kinetic energy, and as is plainly visible in figure 8 above, the RF bunch structure remains relatively intact after the output cavity.

\section{FUTURE DIRECTIONS}

Several areas remain to be covered. The electron gun design, although consistent with electron guns that have been or are currently operating $(17,18,24,26)$, must be addressed. The possibility of tailoring the beam impedance to vary commensurately with the gap impedance of the cavities must also be addressed, and the complexities involved weighed against the elaborate output cavity coupling schemes required to maintain a high degree of field flatness. Once decided, the multi-cell, travelling or standing wave output structure must be designed.

Issues of klystron stability against charge density fluctuations and structure misalignment must be addressed to establish alignment tolerances on the RF cavities relative to the drift tubes. Simulations indicate that charge centroid asymmetries (equivalent to a net displacement of the cavity off the klystron centerline) on of order 1 micron do not induce oscillation. Studies of beam-asymmetry induced coupling to higher modes of the cavity will also have to be examined closely.

More exotic issues to address include the design of the quasi-optical output combining optics for adding together the output of several SBKs to achieve the power levels desired, and the possibility of performing the output power combination in concert with binary pulse compression to produce short, high power pulses.

\section{CONCLUSIONS}

A fully three-dimensional simulation incorporating the beam transport, cavity, and power in/out coupling schemes in a manner consistent with a realizable tube has been presented. Although the gain and efficiency for the particular case shown are less than exciting, the causes of the poor performance are understood, and easily remedied. Given the long simulation running times (days), the Magic3D model is clearly an analysis tool rather than a synthesis tool, but provides the demonstrated ability to model the performance of a sheet beam klystron in full three-dimensional detail.

The three major physics issues unique to the sheet beam klystron, those of beam edge erosion, poor beam cavity coupling, and drift tube oscillations have been brought under control under the fairly ideal circumstances presented here. Thorough numerical 
modeling studies under less ideal circumstances will indicate whether more aggressive measures, such as the inclusion of choke cavities(23) in the drift tubes [to suppress oscillations], are necessary.

\section{ACKNOWLEDGEMENTS}

The authors gratefully acknowledge many illuminating discussions with Roger Miller, Daryl Sprehn, Glenn Scheitrum, Robert Siemann, David Whittum, and Perry Wilson.

\section{REFERENCES}

1. P. B. Wilson, AIP Conf. Proc. 397, 191 (1997).

2. R. H. Siemann, "Advanced Electron Linacs", in Proc. Symp. Elec. Lin. Accel., Stanford, CA, SLAC-R-526, (1998).

3. D. H. Whittum, "Switched Matrix Accelerator", ARDB-86, (1997).

4. G. Caryotakis, "Development of X-band Klystron Technology at SLAC", in Proc. of the Part. Accel. Conf., Vancouver, B.C., (1997).

5. D. N. Smithe, M. Bettenhausen, L. Ludeking, "3-D Simulations of Multiple Beam Klystrons", in these proceedings (1998).

6. E. P. Eppley, SLAC Memorandum dated July 1, 1986.

7. D. U. L. Yu, J. S. Kim, P. B. Wilson, "Design of a High-Power Sheet Beam Klystron", in Proc. IEEE Part. Accel. Conf., Washington D.C., March 16-19, (1997).

8. K. R. Eppley, W. B. Hermannsfeldt, R. H. Miller, "Design of a Wiggler-Focussed, Sheet Beam X Band Klystron”, in Proc. of the IEEE Part. Accel. Conf., Washington D. C., March 16-19, (1997).

9. H. H. Meinel, "Commercial Applications of Millimeterwaves: History, Present Status, and Future trends", IEEE MTT, 43, (7), p. 1639-53, (1995).

10. S. V. Robertson, G. M. Rebeiz, "Micromachined W-Band Filters”, IEEE MTT, 44, (4), p. 598-606, (1996).

11. D. Rutledge, "Micro-machining in Submillimeter-Wave Circuits", IEEE MTT-S Digest, p. 1889-92, (1996).

12. W. R. Fowkes, "Cavity Resonator Measurements at 90 GHz", ARDB-164, (1997). 13. D. T. Palmer, to be published in Proc. Adv. Accel. Conc. Conf., Baltimore, MD, (1998).

14. J. Song, "Deep X-ray Lithography Fabrication of MM-Wave Cavities at the Advanced Photon Source", in Proc. of XIXth LINAC Conf., Chicago, IL, (1998).

15. R. Merte, R. Apel, "S-parameter Measurement of W-band Muffin-tin WBAND003”, TET-NOTE 98/06, (1998).

16. J. H. Booske, B. D. McVey, T.M. Antonsen, "Stability and Confinement of Nonrelativistic Sheet Electron Beams with Periodic Cusped Magnetic Focusing”, $J$. Appl. Phys., 73, (9), p. 4140-55, (1993). 
17. Z. Zhang, et al, "Experimental and Numerical Studies of Sheet Electron Beam Propagation through a Planar Wiggler Magnet", IEEE Tran. Plasma Sci., 21, (6), p. 760-7, (1993).

18. J. H. Booske, et al, "Propagation of Wiggler Focussed Relativistic Sheet Electron Beams", J. Appl. Phys., 64, (1), p. 6-11, (1988).

19. S. Solyga, "Two-Dimensional Design of a Low-Voltage mm-Wave Sheet Beam Klystron", TET-NOTE 98/01, (1998).

20. R. Miller, private communication, (1998).

21. K. H. Kreuchen, A. A. Auld, N. E. Dixon, "A Study of the Broadband Frequency Response of the Multicavity Klystron Amplifier”, J. Elect., p 529-67, (1957). 22. P. Lapostolle, "Proton Linear Accelerators: A Theoretical and Tistorical introduction", LA-11601-MS, (1989).

23. D. Yu, P. B. Wilson, "Sheet-Beam Klystron RF Cavities", in Proc. IEEE Part. Accel. Conf, Washington, D. C., p 2681-3, (1993).

24. D. J. Radack, J. H. Booske, Y. Carmel, W. W. Destler, "Wiggler Focussed Relativistic Sheet Beam Propagation in a Planar Free-Electron Laser Configuration", Appl .Phys. Lett., 55, (20), p. 2069-71, (1989).

25. G. Caryotakis, "The Klystron: A Microwave Source of Surprising Range and Endurance", SLAC-PUB-7731, (1998).

26. V. L. Granatstein, et al, "Near-Millimeter Free Electron Lasers with Small period Wigglers and Sheet Electron Beams", NIM, A272, p.110-16, (1988). 